\title{
Extensive Spinal Cord Injury following Staphylococcus aureus Septicemia and Meningitis
}

\author{
Nicolas De Schryver ${ }^{\mathrm{a}}$ Guy Cosnard ${ }^{\mathrm{b}}$ Vincent van Pesch ${ }^{\mathrm{c}}$ \\ Catherine Godfraind $^{d}$ Philippe Hantson $^{a}$ \\ Departments of antensive Care, ${ }^{\mathrm{b}}$ Neuroradiology and ${ }^{\mathrm{c}}$ Neurology, and \\ dLaboratory of Neuropathology, Cliniques St-Luc, Université catholique de \\ Louvain, Brussels, Belgium
}

\section{Key Words}

Bacterial meningitis · Septicemia $\cdot$ Spinal cord injury $\cdot$ Vasculitis

\begin{abstract}
Bacterial meningitis is rarely complicated by spinal cord involvement in adults. We report a case of Staphylococcus aureus septicemia complicated by meningitis and extensive spinal cord injury, leading to ascending brain stem necrosis and death. This complication was investigated by magnetic resonance imaging which demonstrated intramedullary hyperintensity on T2-weighted images and by multimodality evoked potentials. Postmortem microscopic examination confirmed that the extensive spinal cord injury was of ischemic origin, caused by diffuse leptomeningitis and endarteritis.
\end{abstract}

\section{Introduction}

Bacterial meningitis is rarely complicated by spinal cord injury. We describe a fatal case of Staphylococcus aureus septicemia complicated by meningitis and extensive spinal cord injury, leading to ascending brain stem necrosis. Repeated magnetic resonance imaging (MRI) and a postmortem examination were performed.

\section{Case Report}

A 62-year-old woman who had a history of type 2 diabetes mellitus treated by repaglinide was admitted to the hospital for atypical chest pain. There was no evidence of coronary disease at coronary angiography. During her short hospital stay, the patient developed a superficial phlebitis of the left forearm on the site of the venous perfusion. Two days later, when already discharged, she developed backaches and fever. She was readmitted 4 days later to another hospital with the same complaints. 
At admission, the patient's Glasgow Coma Scale score was $15 / 15$, she was fully alert and had no motor deficit. No neck stiffness was noted at physical examination. The admission laboratory investigations revealed a major inflammatory syndrome (C-reactive protein level of $47.8 \mathrm{mg} / \mathrm{dl}$; normal range $<1 \mathrm{mg} / \mathrm{dl}$ ), with a normal white blood cell count. In addition, renal function was mildly impaired, serum creatinine level was $2.1 \mathrm{mg} / \mathrm{dl}$ (normal range $0.6-1.4 \mathrm{mg} / \mathrm{dl}$ ) and blood urea nitrogen was 84 $\mathrm{mg} / \mathrm{dl}$ (normal range $15-50 \mathrm{mg} / \mathrm{dl}$ ). There was no evidence of a pulmonary or urinary infection. However, an empirical antimicrobial therapy with amoxicillin and clavulanic acid was initiated. Initial blood cultures ( 6 bottles) grew S. aureus. Antimicrobial therapy was changed to vancomycin and then switched to oxacillin ( $2 \mathrm{~g}$ intravenous every $4 \mathrm{~h}$ ) according to the results of the antibiogram. Corticosteroids were not prescribed.

Within 2 days, the patient developed dysarthria with drowsiness and polypnea, and was transferred to the intensive care unit. She became confuse and disorientated, with a stiff and painful neck. Clinical examination of the cranial nerves and peripheral reflexes was normal, and no motor deficit could be observed. Lumbar puncture revealed a purulent cerebrospinal fluid (CSF). The white blood cell count was $31,800 / \mathrm{mm}^{3}$ (normal range $<5,000 / \mathrm{mm}^{3}$ ) with $85 \%$ neutrophils, $10 \%$ lymphocytes and $5 \%$ monocytes. The CSF glucose was $54 \mathrm{mg} / \mathrm{dl}$ and lactate $13.5 \mathrm{mmol} / \mathrm{l}$ (normal range $1-2.2 \mathrm{mg} / \mathrm{dl}$ ). Simultaneous blood glucose determination was not available. The CSF culture remained negative. Contrast-enhanced brain computed tomography (CT) did not reveal any specific lesion. In particular, there was no sign of ventriculitis or venous thrombosis.

The patient was referred to a third hospital (day 14 after the first hospital admission), where she was found to have a Glasgow Coma Scale score of 11/15 (E4V2M5). After intubation, mechanical ventilation was started due to the onset of a major hypoxemia. A magnetic resonance imaging (MRI) of the brain and spinal cord was performed (fig. 1). The entire cervical spinal cord was enlarged, and T2-weighted images showed intramedullary hyperintensity. Diffuse leptomeningeal and focal intramedullary gadolinium enhancements were observed on T1-weighted images (fig. 2). The signal abnormalities extended to the medulla oblongata. No significant changes were identified in the cerebral hemispheres and, in particular, there was no evidence of hydrocephalus or brain edema. Brain stem auditory evoked potentials (BAEPs) were preserved. In contrast, upon median nerve stimulation, no cortical somatosensory evoked potentials (SSEPs) were recorded. Peripheral activities (P9 and N13 complex) were normal. The absence of the P14 complex, thought to be generated by the medial lemniscus, indicated damage caudal to the bulbomedullary junction.

The patient's neurological condition worsened within 2 days. Spontaneous breathing disappeared, and the patient had to be ventilated in a pressure control mode. Dysautonomic episodes with hypotension and extreme bradycardia were noted. Complete quadriparesis and anesthesia were observed, together with horizontal ophthalmoplegia. The patient was conscious and able to respond to simple questions by vertical eye movements. Blood cultures were still positive for methicillin-sensitive $S$. aureus 1 week after the initiation of an adequate antimicrobial therapy. Endocarditis could be ruled out by transesophageal echocardiography. MRI was repeated on day 19 and revealed that the signal hyperintensity extended further from the medulla and the cerebellar tonsils to the thoracic spinal cord. Upon T1-weighted imaging, signal hypointensity of the grey matter suggested necrotic damage. On day 21 , all the brain stem reflexes were absent, as were BAEPs, except for a persisting wave I upon left ear stimulation, indicating ascendant pontine damage.

Due to the extensive anatomical lesions and the presence of dysautonomic complications including bradycardia and hypotension, it was decided, together with the patient's relatives, to withdraw intensive care therapy. The patient died from cardiocirculatory failure, and an autopsy was performed. Macroscopic and microscopic examination confirmed the diagnosis of extensive leptomeningitis with vasculitis and secondary medullar ischemia (fig. 3 ). No other metastatic area of the S. aureus infection was found.

\section{Discussion}

Bacterial meningitis is rarely complicated by spinal cord involvement and, according to the literature, children are more frequently affected than adults [1-7]. The spinal cord may be injured by compression due to a spinal abscess, by ischemia due to vasculitis, shock, herniation, arachnoiditis, or by myelitis [2]. 
The causative germs are mainly Neisseria meningitidis, Streptococcus pneumoniae, Escherichia coli, and Haemophilus influenzae, while the involvement of S. aureus seems exceptional. Our patient initially presented with meningitis (likely caused by $S$. aureus septicemia) but rapidly showed manifestations of brain stem involvement (dysarthria and respiratory failure). Signs of spinal cord injury with quadriparesis were noted afterwards. The clinical progression correlated with the worsening of the MRI findings and the electrophysiological testing. Indeed, deterioration of BAEPs indicated structural pontine damage, and the absence of SSEPs indicated severe damage to the lemniscal pathways caudal to the bulbo-medullary junction.

The nature and extent of spinal cord dysfunction are well investigated by MRI. In a description of 3 adult cases of bacterial meningitis complicated by paraparesis or tetraparesis, Kastenbauer et al. [1] demonstrated hyperintensities on T2-weighted images that predominantly involved the grey matter and extended from the cervical to the lumbar cord. None of the patients showed evidence of an intramedullary abscess or extrinsic cord compression by an epidural hemorrhage, an extramedullary abscess, or a subdural empyema. In all cases, severe paresis persisted, together with bowel and bladder incontinence. Follow-up MRI showed that hyperintensities had resolved in 1 patient and progressed in another, while central cavitation had developed in the cervical spinal cord of the last case.

In our patient, compression by empyema could be ruled out by MRI. The differential diagnosis in MRI was ischemic infarction, myelitis, or a combination of both factors. The prevalence of acute transverse myelitis associated with $S$. aureus is rare. Most of the time, it occurs as a consequence of a hematologic spread from another infected site that can be pulmonary, cardiac, skeletal, genitourinary, gastrointestinal or cutaneous [8]. S. aureus myelitis may also develop from a local infection such as a pyomyositis of the paraspinal muscles [9].

The diagnosis of an infectious myelitis is based on MRI findings, which typically show a spinal cord hypersignal on T2-weighted images, which is sometimes associated with swelling $[1,8]$. The differential diagnosis of an intramedullary spinal cord lesion with a high T2 signal intensity on MRI should also include primary neoplasm and infarction involving the anterior spinal artery. Less common etiologies include recent trauma, multiple sclerosis, sarcoidosis, HIV infection, systemic lupus erythematosus, radiation therapy, exposure to toxins, vascular malformation and herpes infection [8].

The pathological findings in the present case excluded the diagnosis of myelitis and confirmed that the spinal cord injury was of ischemic origin. The patient never experienced hypotension or shock, which could have contributed to vascular compromise of the spinal cord. There was no evidence of cerebral septic venous thrombosis. We conclude that the mechanism was likely a decrease in blood supply due to extensive arachnoiditis, which is rarely reported after bacterial meningitis [10-12]. Despite the persistence of positive blood cultures under appropriate antimicrobial therapy, we did not observe any abscess formation. The source of the bacteremia was never definitely identified, but could be a possible peripheral thrombophlebitis.

\section{Disclosure Statement}

The authors have no conflict of interest to declare. 


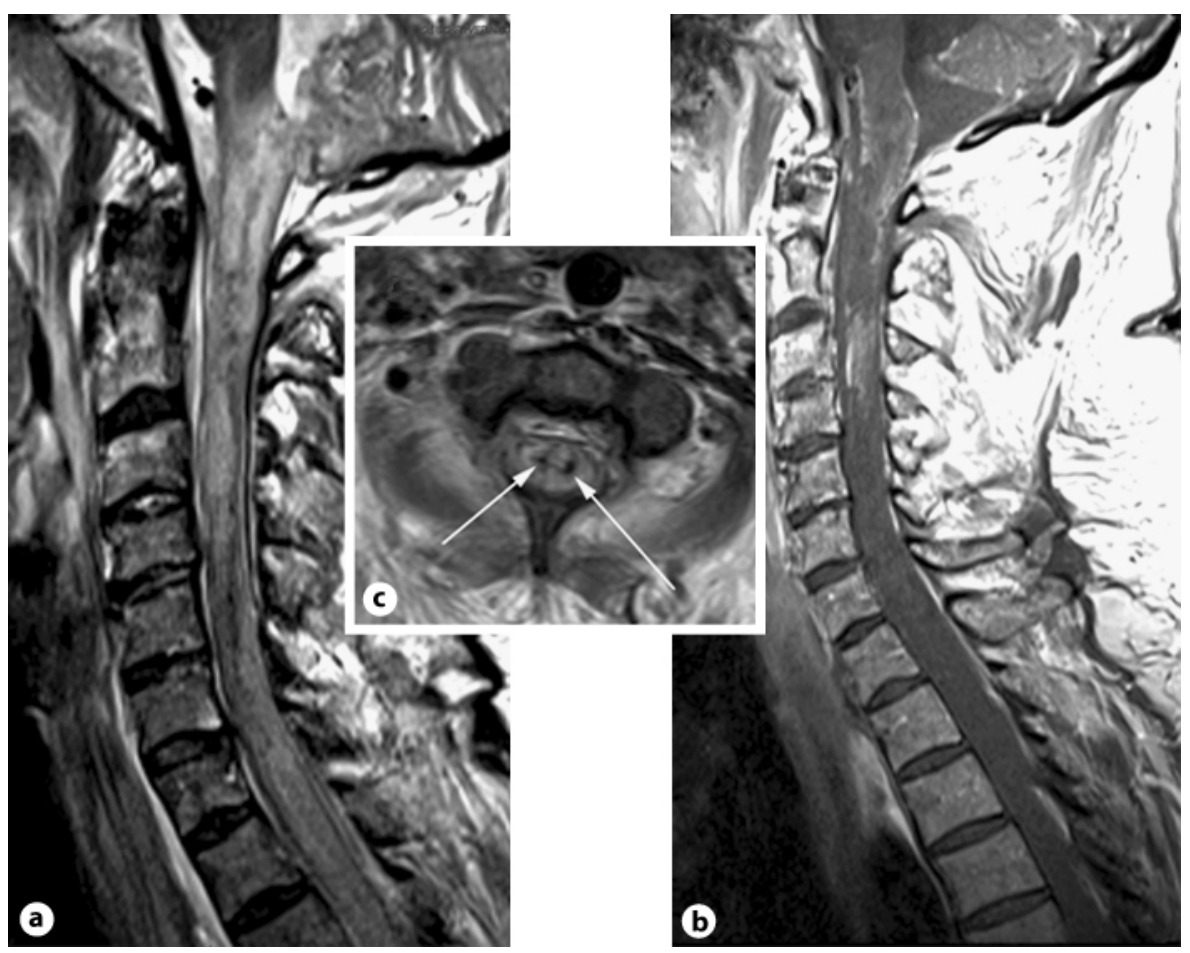

Fig. 1. a Sagittal T2-weighted MR image showing an enlarged spinal cord with heterogeneous signal intensity, indicative of edema and hemorrhage. b Sagittal T1-weighted MR image following intravenous gadolinium administration, showing marked diffuse leptomeningeal and discrete focal spinal cord enhancements. c Axial T2-weighted MR image showing hemorrhagic foci with low signal intensity in the grey matter (arrows). 

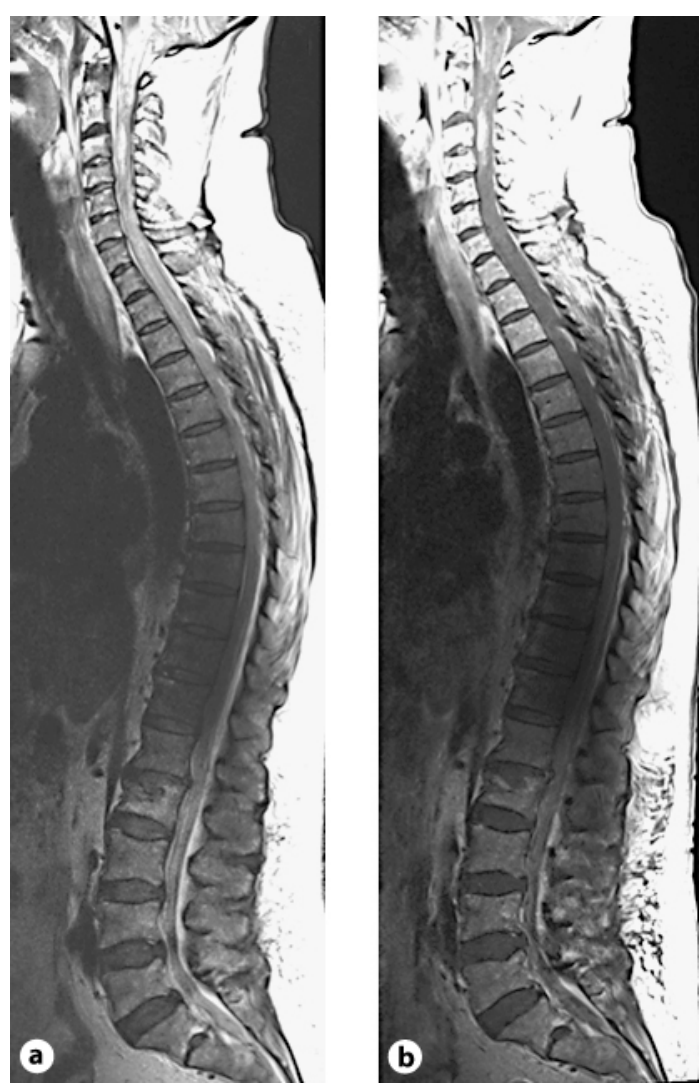

Fig. 2. Total spinal cord view. Sagittal T2-weighted (a) and T1-weighted (b) MR images following intravenous gadolinium administration. 


\begin{tabular}{c|l|l|l}
$\begin{array}{c}\text { Case Reports in } \\
\text { Neurology }\end{array}$ & $\begin{array}{l}\text { Case Rep Neurol 2011;3:147-153 } \\
\text { DOl: } 10.1159 / 000329841\end{array}$ & $\begin{array}{l}\text { Published online: } \\
\text { June 27, 2011 }\end{array}$ & $\begin{array}{l}\text { ○ 2011 S. Karger AG, Basel } \\
\text { ISSN 1662-680X } \\
\text { www.karger.com/crn }\end{array}$ \\
\hline
\end{tabular}

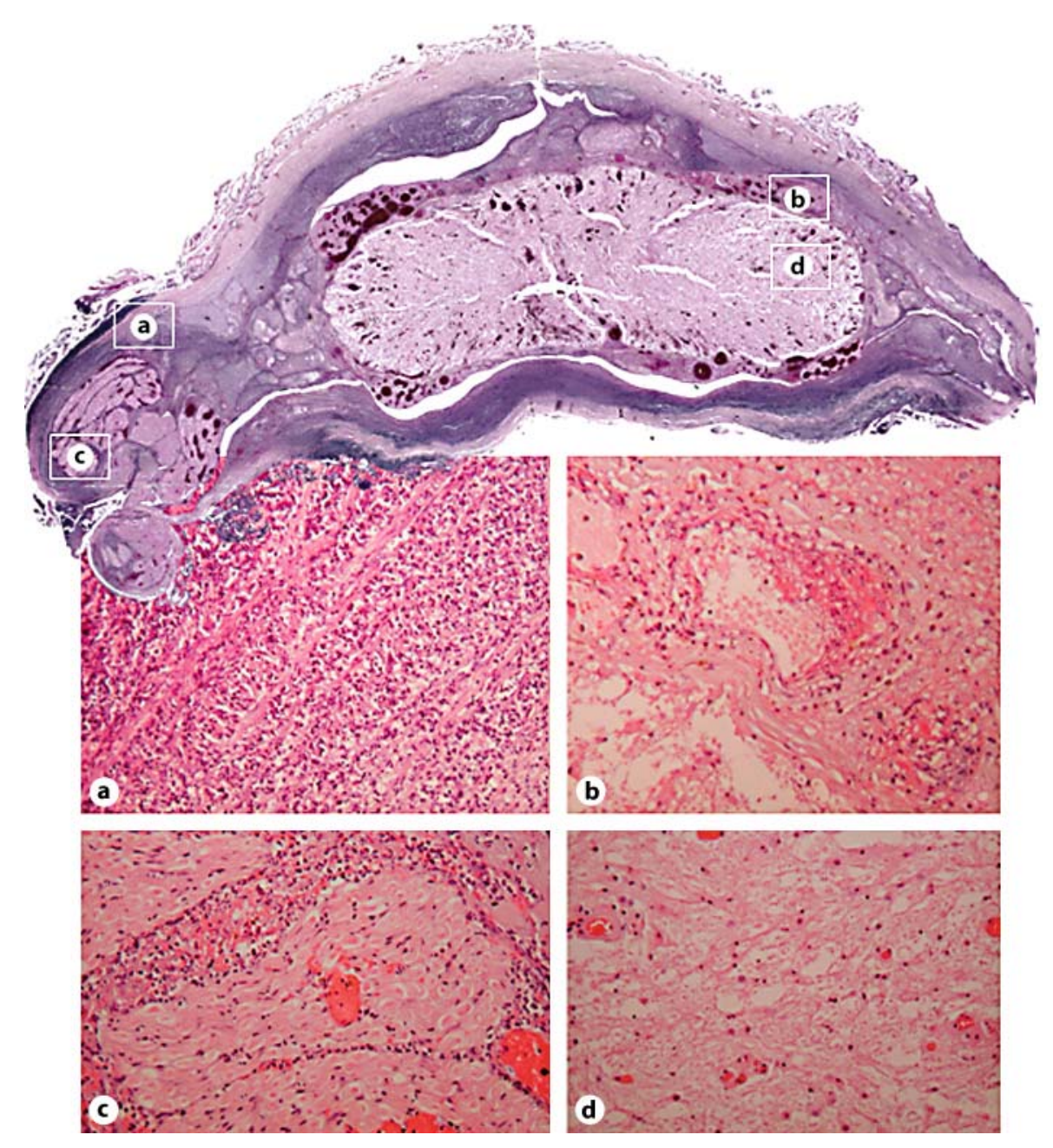

Fig. 3. HE-stained cross-section of the spinal cord demonstrating diffuse inflammatory infiltrates of the leptomeninges (a) and spinal roots (c). Infiltrates were mostly composed of polymorphonuclear cells $(\mathbf{a}-\mathbf{c})$, inducing endarteritis $(\mathbf{b})$ and secondary parenchymal infarction $(\mathbf{d})$.

\section{References}

-1 Kastenbauer S, Winkler F, Fesl G, Schiel X, Ostermann H, Yousry TA, Pfister HW: Acute severe spinal cord dysfunction in bacterial meningitis in adults: MRI findings suggest extensive myelitis. Arch Neurol 2001;58:806-810.

2 Moffett KS, Berkowitz FE: Quadriplegia complicating Escherichia coli meningitis in a newborn infant: case report and review of 22 cases of spinal cord dysfunction in patients with acute bacterial meningitis. Clin Infect Dis 1997;25:211-214.

3 Kikuchi M, Nagao K, Muraosa Y, Ohnuma S, Hoshino H: Cauda equina syndrome complicating pneumococcal meningitis. Pediatr Neurol 1999;20:152-154.

-4 Bouygues D, Sanmarti F, Ponsot G, Auberge C, Arthuis M: Myélopathies aiguës au cours des méningites purulentes. Arch Fr Pediatr 1980;37:103-107.

5 Puvabanditsin S, Wojdylo EW, Garrow E, Kalavantavanich K: Group B streptococcal meningitis: a case of transverse myelitis with spinal cord and posterior fossa cysts. Pediatr Radiol 1997;27:317-318.

-6 Mathew P, Todd NV, Hadley DM, Adams JH: Spinal cord infarction following meningitis. Br J Neurosurg 1993;7:701-704. 
-7 O'Farrell R, Thornton J, Brennan P, Brett F, Cunningham AJ: Spinal cord infarction and tetraplegia - rare complications of meningococcal meningitis. Br J Anaesth 2000;84:514-517.

-8 Friess HM, Wasenko JJ: MR of staphylococcal myelitis of the cervical spinal cord. AJNR Am J Neuroradiol 1997;18:455-458.

-9 Kulkarni GB, Pal VK, Veena Kumari HB, Goyal M, Kovoor JM, Nadig S, Arakere G: Community-acquired methicillin-resistant Staphylococcus aureus pyomyositis with myelitis: a rare occurrence with diverse presentation. Neurol India 2009;57:653-656.

10 Turner JW: Spinal cord lesions in cerebrospinal fever. Lancet 1948;1:398-402.

11 Haupt HM, Kurlinski JP, Barnett NK, Epstein M: Infarction of the spinal cord as a complication of pneumococcal meningitis. Case report. J Neurosurg 1981;55:121-123.

12 Van Egeraat SC, Van Munster ET, Bodewes HW, Van der Hoeven JG: Spinal cord infarction as a severe complication of meningococcal meningitis. Eur J Intern Med 2003;14:383-385. 